\title{
Prosthetic valve endocarditis precipitating bleeding from small bowel angiodysplasia
}

\author{
Perera GND ${ }^{1}$, Kulatunga A $\mathbf{A}^{1}$, Samarasekera $\mathbf{D N}^{2}$ \\ ${ }^{I}$ National Hospital of Sri Lanka, ${ }^{2}$ Department of Surgery, Faculty of Medicine, University of Colombo, \\ Sri Lanka
}

Correspondence: Dr. G.N.D. Perera; (nilanka.dushani@gmail.com)

\section{Introduction}

There are a few case reports of angiodysplasia of gastrointestinal tract associated with aortic stenosis. This rare association is known as Heyde syndrome. We illustrate a case of angiodysplasia in the jejunum associated with aortic stenosis in a 73 year old man with prosthetic valve endocarditis. In our patient, infective endocarditis episode was associated with severe bleeding from small bowel angiodysplasia.

\section{Case History}

A 73-year-old retired bank manager who had undergone bioprosthetic aortic valve replacement in 2008 for severe aortic stenosis presented 2 years later with aphasia and right sided hemiparesis. He had a history of iron deficiency anaemia due to occult gastrointestinal blood loss.

Iron deficiency had been diagnosed in 2006 when he presented with symptoms of severe anaemia requiring blood transfusions. Upper gastrointestinal endoscopy (UGIE) and lower gastrointestinal endoscopy (LGIE) did not reveal a bleeding site. However, occult blood was detected in stool repeatedly. As small bowel endoscopy procedures were unavailable during this period, cause for the occult gastrointestinal bleeding remained a diagnostic problem. He was continued on oral iron replacement therapy with a poor response and required multiple blood transfusions until he underwent bioprosthetic aortic valve replacement with coronary artery bypass graft $(\mathrm{CABG})$ in 2008 .
During the next 2 years following the valve replacement, our patient remained free of anaemia. On this admission, he presented with a cerebral thromboembolic episode due to prosthetic valve endocarditis. He had elevated inflammatory markers with a positive blood culture for $\alpha$ haemolytic streptococci. His haemoglobin was $6.2 \mathrm{~g} / \mathrm{dL}$ with a hypochromic microcytic picture compatible with iron deficiency. There was no microangiopathic haemolysis detected. Coagulation profile was normal. The transoesophageal echo (TOE) revealed a vegetation of $0.2 \times 0.3 \mathrm{~cm}$ attached to the aortic valve prosthesis. The patient was given several blood transfusions during the first week to maintain a stable haemoglobin level. But the transfusion requirement ceased following treatment of endocarditis and he was continued on iron supplements thereafter.

As the patient improved, we re-evaluated his anaemia with repeat endoscopies. UGIE and LGIE were both normal. We proceeded on to capsule endoscopy to evaluate for a possible small bowel source for blood loss. Capsule endoscopy recordings over $24 \mathrm{hrs}$ revealed 3 sites of angiodysplasia in the proximal jejunum (Figure).

There was no evidence of active bleeding at the time of endoscopy. Our patient was considered to have Heyde syndrome which remained quiescent following valve replacement but rebled with the development of prosthetic valve endocarditis. 


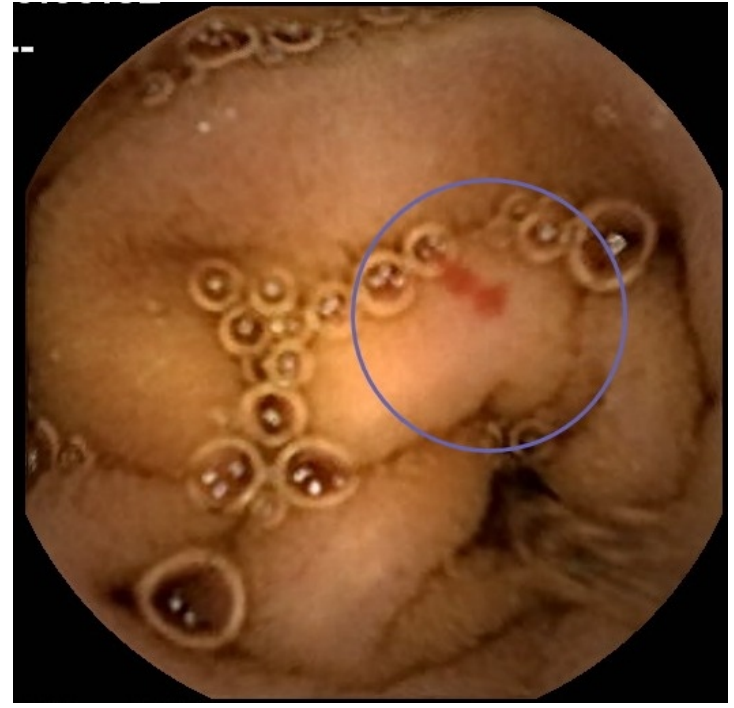

Figure: Angiodysplasia of proximal jejunum in capsule endoscopy

\section{Discussion}

Our patient had severe aortic stenosis requiring valve replacement. He also had occult gastrointestinal bleeding from small bowel angiodysplasia requiring multiple blood transfusions and iron replacement. Association of aortic stenosis with gastrointestinal tract angiodysplasia is known as Heyde syndrome. It was first described by Dr Edward C Heyde in 1958(1). Since then many authors have described the possible association (2).

There are doubts whether both conditions co exist as a common manifestation in the elderly or whether low grade mucosal ischaemia in aortic stenosis predisposes to angiodysplasia of gut(3). In recent reports, a coagulation defect was also identified in these patients with aortic stenosis leading to increased bleeding from the gut angiodysplasia. High shear stress across the stenosed valve results in damage to Von Willebrand Factor (vWF) multimers causing an acquired Von Willebrand disease (vWD) type 2A (4). Interestingly, bleeding ceases in these patients following aortic valve replacement. This finding is supported by many case reports (3) in literature and valve replacement is considered as a treatment option for bleeding angiodysplasia in Heyde syndrome. Our patient had similar findings with gastrointestinal bleeding being quiescent for the last 2 years following valve replacement. But he had rebleeding from small bowel angiodysplasia with the development of endocarditis. Whether infective endocarditis could exacerbate consumptive coagulopathy in Von Willebrand disease (vWD) is still unknown. But it is important to note that anaemia in infective endocarditis associated with aortic stenosis could be partly due to bleeding angiodysplasia as in our index case. Hence it is worthwhile to look for a possible gastrointestinal source of blood loss in such a circumstance.

Treatment of these patients with combined oestrogen and progesterone therapy has reduced transfusion requirements by half (3). Endoscopy with argon beam diathermy and bowel resection has been evaluated. However, a proportion of patients could be managed with supportive therapy as our patient.

\section{Conclusion}

Aortic stenosis is associated with bleeding from gastrointestinal angiodysplasia due to acquired Von Willebrand Disease type 2A (vWD). It is worthwhile to look for a possible gastrointestinal source of blood loss in patients with iron deficiency anemia in infective endocarditis associated with aortic stenosis.

\section{References}

1. Heyde EC. Gastrointestinal bleeding in aortic stenosis. New England Journal of Medicine 1958; 259: 456.

2. Pate GE, Mulligan A. An Epidemiological Study of Heyde's Syndrome: An Association between Aortic Stenosis and gastrointestinal bleeding. The Journal of Heart Valve Disease 2004; 13: 713-16.

3. Hasan F, O'Brien CS, Sanyal A, Dalton HR. Aortic stenosis and gastrointestinal bleeding. Journal of Royal Society of Medicine 2004;97(2): 81-2.

4. Yoshida K, Tobe S, Kawata M, Yamaguchi M. Acquired and reversible von Willebrand disease with high shear stress aortic valve stenosis. Annals of Thoracic Surgery 2006; 81:490-4.

5. Van Cutsem E, Rutgeerts P, Vantrappen G. Long-term effect of hormonal therapy for bleeding gastrointestinal vascular malformation. European Journal of Gastro Hepatology 1993; 5: 439-43. 